\title{
Auswirkungen der COVID-19-Krise auf die Struktur und den Inhalt von IT-Management-Stellenanzeigen
}

\author{
Bjarne Erik Roscher
}

Eingegangen: 1. Februar 2021 / Angenommen: 19. April 2021 / Online publiziert: 26. Mai 2021

(C) Springer Fachmedien Wiesbaden GmbH, ein Teil von Springer Nature 2021

Zusammenfassung Unternehmen sind immer mehr auf Informationstechnologie (IT) angewiesen, und das Schlagwort Digitalisierung ist überall präsent. Einige Unternehmen erfinden neue Geschäftsmodelle, die auf disruptiven Innovationen basieren, welche von Informationstechnologie unterstützt werden. Andere passen ihre Geschäftsmodelle als Follower an. Alle diese neuen Geschäftsprozesse und die zugrunde liegende Informationstechnologie müssen von IT-Organisationen gestaltet, gewartet und unterstützt werden. Die Covid-19-Pandemie im Jahr 2020 forderte Unternehmen und Organisationen auf verschiedene Arten heraus. Die wichtigste Veränderung ist, dass Arbeitnehmer im Homeoffice arbeiten mussten, während sie versuchten, den Geschäftsbetrieb aufrechtzuerhalten. Dies führte in einigen Unternehmen zu einem Digitalisierungsschub in Bezug auf flexibles Arbeiten. Um von außerhalb des Firmengeländes arbeiten zu können, musste in einigen Fällen neue IT-Infrastruktur aufgebaut und die Anpassung von Geschäftsprozessen durchgeführt werden. In der aktuellen Studie werden Stellenanzeigen analysiert, um herauszufinden, ob sich die Covid-19-Krise auf den Fokus und die Prioritäten der IT-Abteilungsleiter ausgewirkt hat. Die Annahme dieser Studie ist, dass sich der Inhalt neuer Stellenangebote durch den Einfluss von COVID-19 ändern würde. Als Forschungsmethode wurde eine strukturierte Textanalyse von IT-Top-Management Stellenanzei-

\footnotetext{
Hinweis Dieser Artikel wurde ursprünglich in englischer Sprache auf der 3rd International Conference on Research in Business, Management and Economics in Dublin, Irland präsentiert und in den Proceedings veröffentlicht (Bjarne Erik Roscher 2020).
}

\footnotetext{
Bjarne Erik Roscher $(\bowtie)$

Department of Business, Management and Economics, Universität von Lettland, Riga, Lettland E-Mail: bjarne@roscher.net

FOM Hochschule für Oekonomie \& Management, Standort Nürnberg, Essen, Deutschland

Technische Hochschule Nürnberg, Georg Simon Ohm, Nürnberg, Deutschland

Hochschule für angewandte Wissenschaften Würzburg-Schweinfurt, Würzburg, Deutschland
} 
gen in Deutschland gewählt. Um Unterschiede vor und während der Pandemiekrise abzubilden, wurden Daten zu zwei verschiedenen Zeitpunkten im Jahr 2020 gesammelt. Nach dem Vergleich der codierten Schlagwörter in beiden Datensätzen ist in der Analyse keine signifikante Änderung der Anforderungen von Aufgaben oder der Struktur der Stellenanzeigen festzustellen. Dies führt zu der Schlussfolgerung, dass die Personalabteilungen in Unternehmen überwiegend vorhandene Templates nutzen und bei der Erstellung von Stellenanzeigen nicht ausreichend veränderte Rahmenbedingungen einarbeiten. Die einzige Spur, die COVID-19 in Stellenanzeigen hinterlassen hat, sind Hinweise auf Home-Office-Richtlinien.

Schlüsselwörter Chief Information Officer (CIO) · IT Organisation • Personalbeschaffung · COVID-19 Krise

\title{
Impact of the COVID-19 Crisis on the Structure and Content of Job Advertisements
}

\begin{abstract}
Companies are more and more dependent on information technology (IT) and the buzzword digitization is present everywhere. Some companies are inventing new business models based on disruptive innovation supported by information technology. Others adapt these business models as followers. All these new business processes and underlying information technology need to be managed, serviced, and supported by IT organizations to ensure business continuity. Due to the Covid19 pandemic in the year 2020, companies and organisations have been challenged with the fact that workers had to stay and work at home while trying to keep the operations running. This led in some companies to a digitization boost in terms of remote working, which needed new IT-Infrastructure and the adoption of business processes to function from outside the company property. In the current research, job advertisements have been analysed to investigate if the Covid-19 crisis had an impact on the focus and priorities of IT department heads. The initial assumption this research is based on is, that the content of new job openings posted on recruiting webpages in Germany would change through the influence of COVID-19. The research method was a structured content analysis of job offerings looking for IT top management positions in Germany. Data was collected at two different times in the year 2020 to reflect differences before and within the pandemic crisis. After comparing the coded keywords in both datasets no significant change in requirements of tasks or structure of the job ads can be seen in the analysis. Which leads to the conclusion that recruiting departments within companies do not really collect requirements for top management IT positions through alignment with the business. The only traces the COVID-19 has left in the job adds is that in some cases homeoffice policies are mentioned.
\end{abstract}

Keywords Chief Information Officer (CIO) - IT Director · IT Organization • Recruitment · Covid-19 cisis 


\section{Auswirkungen vor und während COVID-19 auf IT-Lösungen und ihr Management}

Synnott definierte 1981 die Rolle des Chief Information Officer (CIO) als ,Senior executive responsible for establishing corporate information policy, standards, and management control over all corporate information resources" (Synnott und Gruber 1981). Diese Berufsbezeichnung wurde auch in anderen Ländern übernommen, darunter in Deutschland. Diese Studie zeigt jedoch, dass dies hauptsächlich in größeren Unternehmen der Fall ist, während kleine und mittelständische Unternehmen (KMU) ihre IT-Führungskräfte „IT-Leiter“, „IT-Manager“ oder „IT-Abteilungsleiter“ nennen. Untersuchungen aus den USA zeigen, dass andere Berufsbezeichnungen wie „IT Director“, „SVP/VP IT“ und „IT Manager“ auch dort weit verbreitet sind (Tallon 2014).

Viele Studien zeigen die Wichtigkeit der Rolle eines CIO für ihre Organisation.

So kann ein CIO die Unternehmensleistung positiv beeinflussen, indem er die ITEffizienz fördert, was bedeutet, die Kosten zu senken und dadurch den Gewinn zu steigern. Ebenso wichtig ist es auch die IT-Effektivität durch eine verbesserte ITNutzung zu erhöhen (Durst 2007).

Maßnahmen zur Steigerung der IT-Effizienz sind die Einführung maßgeschneiderter interner IT-Managementsysteme (Schweda und Schmidt 2014), effizienter ITService-Management-Prozesse (ITSM) (Marrone und Kolbe 2011) und der passende organisatorische Aufbau (Hodgkinson 1992). Von wesentlicher Bedeutung ist ein effizienter Budget- und Controlling Prozess, der sicherstellt, dass Investitionen die geplanten Ergebnisse liefern (Chan et al. 2006). Maßnahmen zur Verbesserung der IT-Effektivität unterstützen Geschäftsprozesse mit geeigneter Informationstechnologie (Tallon 2014).

Letztendlich besteht die Hauptaufgabe eines CIO darin, das „Business-IT-Alignment“ (BITA) sicherzustellen. Dies führt zu strategischen IT-Initiativen, die die Unternehmensleistung steigern (Peppard 2010). Größere externe Ereignisse beeinflussen den IT-Strategieprozess und müssen in Bezug auf Auswirkungen erfasst und in die IT-Strategie einbezogen werden (Ward und Peppard 2002).

Frühere Forschungen haben den Einfluss des externen Umfelds auf Unternehmen und den Einsatz von Informationstechnologie gezeigt. So kann der strategische Einsatz von Informationstechnologie zur Bewältigung von Herausforderungen in der Unternehmensumwelt beitragen (Kim und Jee 2007; King et al. 1989).

\subsection{Literatur zum Thema Digitalisierung in Zeiten von COVID-19}

IT-Management-Forschung, die im Zuge der COVID-19 Krise entstanden ist, beleuchtet verschiedenste Aspekte. Laut Meiler fördert Covid-19 die digitale Transformation (Meiler 2020). Kumar beschreibt, wie der Einsatz von „Industrie 4.0“ IT-Anwendungen und -Lösungen im Bereich der Einzelhandelslieferkette der Branche helfen kann die Herausforderungen zu meistern (Kumar et al. 2020b). Chen sucht die Bereitschaft der Kunden, für IT-Lösungen zu bezahlen, welchen ihnen bei der Bewältigung von Herausforderungen des täglichen Lebens in Zeiten der Pandemie helfen könnten (Chen et al. 2020). Kumar schlägt vor, wie das Produkti- 
onsmanagement in Zeiten von Corona nachhaltig organisiert werden kann (Kumar et al. 2020a). Und schließlich geben Dwivendi et. al. einen sehr komprimierten und dennoch umfassenden Überblick über Herausforderungen im Bereich des Informationsmanagements. Es handelt sich bei der Arbeit um eine Zusammenstellung von Erkenntnissen, Forschungsergebnissen und Empfehlungen (Dwivedi et al. 2020).

\subsection{Literatur und weitere Quellen zur Eingrenzung des Forschungsvorhabens}

Aufgrund der Aktualität des Themas fand die Literaturrecherche nur wenige wissenschaftliche Arbeiten zum Thema IT-Management und IT-Rekrutierung in der Covid-19-Krise. Eine solche Krise mit Auswirkungen auf so viele Bereiche wie Medizin, Soziales, Wirtschaft, Organisation, Verfahren, Verwaltung sowie auf den persönlichen Bereich hat es in der modernen Welt in dieser Form noch nicht gegeben. Auch der Versuch diese Einschränkungen durch Digitalisierung auszugleichen, hat nie zuvor in diesem Ausmaß stattgefunden. Um den Rahmen für diese Arbeit zu setzen werden Beiträge der IT-Management-Forschung betrachtet (Wollnik 1988; Szyperski und Winand 1989; Krcmar 1991), der IT-Governance Forschung (Weill und Woodham 2002; Rüter et al. 2010; Weill und Ross 2004; Grohmann 2003) sowie der Risikomanagement Forschung (Zellmer 1990). Um die Prioritäten des ITManagements in der aktuellen Krise zu ermitteln, wurde auf nicht wissenschaftliche Veröffentlichungen zurückgegriffen. Unter anderem auf den Bericht zur Lage der IT Sicherheit in Deutschland 2020 vom Bundesamt für Sicherheit in der Informationstechnik (BSI 2020) auf einen Preprint (Mouton und Coning 2020) und Artikel in [nicht wissenschaftlichen] Zeitschriften (Malecki 2020; Barak 2020; Faulds und Raju 2020; Furnell und Shah 2020). In dieser Arbeit wurden jedoch auch einige wenige wissenschaftliche Veröffentlichungen zum allgemeinen Verständnis des ITManagements in Zeiten von COVID-19 berücksichtigt (Dwivedi et al. 2020; Kumar et al. 2020a, b; Chen et al. 2020; Meiler 2020). Der Inhalt dieser Arbeiten wird nicht näher diskutiert, da es sich bei der vorliegenden Arbeit um eine methodisch getriebene Forschung handelt.

\section{Forschungsmethodik}

\subsection{Datenerhebung und -auswahl}

Die Datenerhebung wurde zu folgenden Zeitpunkten durchgeführt:

a) 18.02.2020, einige Tage vor Beginn der COVID-19-Pandemie in Deutschland mit all ihren Maßnahmen des ,,social distancing“ (1. Welle Lockdown) und ihren Auswirkungen auf das Geschäftsleben.

b) 28.10.2020, kurz vor der Einführung der Maßnahmen, um die 2. Welle einzudämmen. Die Quelle für den ersten Datensatz (a) waren die Internet-Jobportale von Indeed.com und Linkedin.com. Die Quelle für den zweiten Datensatz (b) war Indeed.com, da nach der Durchführung von manuellen Stichproben bei LinkedIn keine zusätzlichen Daten gefunden wurden. 

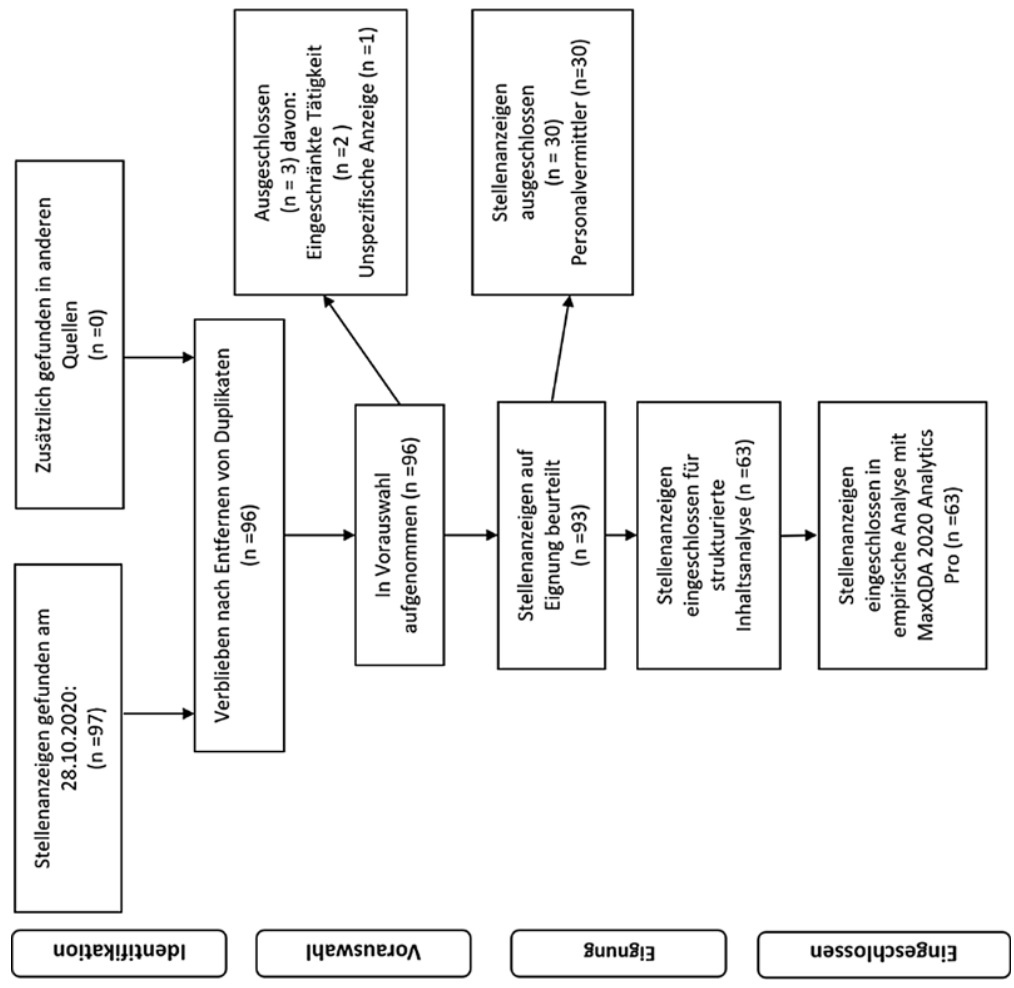

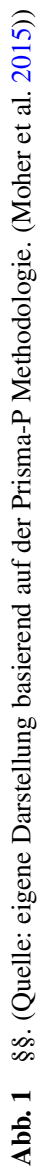


Bei beiden Datenerhebungen wurden alle an diesem Tag verfügbaren Stellenanzeigen mit den Suchbegriffen „IT-Manager“, „IT-Leiter“ und „Chief Information Officer (CIO)“ gespeichert, und anschließend auf Eignung überprüft. Auswahlkriterium war, dass die Stellenanzeigen alle Aspekte eines IT-Managers abdecken. Dazu wurden die Aufgabenbereiche mit dem Modell von Krcmar verglichen (Krcmar 2015). Im ersten Datensatz (a) wurden alle Anzeigen ausgeschlossen, die von einem Personalvermittler veröffentlicht wurden. Der Grund für diesen Ausschluss ist, dass diese in der Regel keine Informationen über den Arbeitgeber enthalten. Im zweiten Datensatz (b) wurden diese trotz der fehlenden Informationen gesammelt, aber später bei der Datenbereinigung ausgeschlossen. Die Auswahl der relevanten Daten wurde gemäß der PRISMA-P-Forschungsmethode (Moher et al. 2015) durchgeführt (siehe Abb. 1).

\subsection{Codierung}

Eine Internetsuche nach Umfragen und Veröffentlichungen von Unternehmensberatungs- und Forschungsunternehmen zum Thema IT Management und COVID-19 lieferte folgende Ergebnisse: PwC (PricewaterhouseCoopers 2020b), Gartner (Shen et al. 2020), KPMG (Bates 2020), Deloitte (2020). Zusätzlich wurden Artikel in relevanten IT-Management-Zeitschriften (Samuels 2020; IDG 2020) verwendet. In diesen Publikationen wurden die fünf wichtigsten Prioritäten identifiziert, die als Maßnahmen im Umgang mit den Herausforderungen der COVID-19-Krise von CIOs genannt werden. Die identifizierten Prioritäten wurden ohne das, in den Umfragen oder Texten verwendete Ranking (falls vorhanden), übernommen. Nach der Verallgemeinerung des Wortlauts wurde eine Frequenzanalyse in der verwendeten Literatur durchgeführt, die zu einem in der Tab. 1 aufgeführten Ranking führte.

Die Prioritäten wurden anschließend in die deutsche Sprache übersetzt und Synonyme, die diese Wörter beschreiben, identifiziert. Da einige Stellenangebote in eng-

Tab. 1 CIO Prioritäten und abgeleitete Beispiele für Suchphrasen. (Quelle: eigene Analyse)

\begin{tabular}{|c|c|c|}
\hline $\begin{array}{l}\text { CIO Prioritäten vor und } \\
\text { während COVID-19 }\end{array}$ & $\begin{array}{l}\text { Rang } \\
\text { (Nennungen) }\end{array}$ & $\begin{array}{l}\text { Beispiele für Suchbegriffe } \\
\text { (sowohl in deutscher als auch in englischer Sprache) }\end{array}$ \\
\hline Digital collaboration & 1. $(n=4)$ & Video Conference, Heimarbeit; Homeoffice etc \\
\hline Information Security & 2. $(n=3)$ & InfoSec, Sicherheit, Angreifer, ISO 2700, etc \\
\hline $\begin{array}{l}\text { Digitization of Business } \\
\text { Model }\end{array}$ & 2. $(n=3)$ & Geschäftsmodel, Business Modell/Case etc \\
\hline IT Strategy and Architecture & 2. $(n=3)$ & Strategie, Architektur, Landschaft, Planung, etc \\
\hline $\begin{array}{l}\text { Customer Interface digital } \\
\text { enhancement }\end{array}$ & 3. $(n=2)$ & Kunden, CRM, etc \\
\hline Data Security & 4. $(n=1)$ & Datenschutz, BDSG, DSGVo, etc \\
\hline Ensure business continuity & 4. $(n=1)$ & Continuity, Aufrechterhaltung, etc \\
\hline Automation & 4. $(n=1)$ & Automatisierung, Digitalisierung \\
\hline Risk Management & 4. $(n=1)$ & Compliance, Risiko \\
\hline Governance & 4. $(n=1)$ & Regeln, Gesetze, Vorschriften, Normen, etc \\
\hline Workforce & 4. $(n=1)$ & Personalplanung, Workforce, Einsatzplanung, etc \\
\hline Cost Control & 4. $(n=1)$ & Einsparung, Controlling, Budget, etc \\
\hline
\end{tabular}


lischer Sprache veröffentlicht wurden, mussten die Suchphrasen zurückübersetzt und synchron mit den deutschen Phrasen verwendet werden. Anhand der Methode von Mayrings zur Inhaltsanalyse, die ursprünglich 1983 (Mayring 2015) veröffentlicht wurde, wurden Paraphrasen verwendet, um die Dokumente nach Sätzen zu durchsuchen, welche von den definierten Suchphrasen umgeben sind. Die gefundenen Sätze enthielten weitere Ausdrücke, die zur Erstellung einer Wortliste verwendet wurden. Diese wurde für die endgültige Suche in allen Stellenangeboten verwendet. Alle gefundenen Sätze wurden manuell überprüft und die Entscheidung für die Aufnahme wurde aufgrund von Attributen zu den Suchphrasen getroffen. Einige Beispiele zeigen, wie Entscheidungen getroffen wurden: „Verantwortlich für“, „,Vorantreiben“ und „Beitragen“ sind Indikatoren für die Aufnahme in die Ergebnisliste. Wohingegen ,durchführen“, „,kontrollieren“, „Interesse an“, „Erfahrung“ Indikatoren für den

Ausschluss sind, da sie darauf hinweisen, dass die entsprechende IT-Führungskraft keine treibende, sondern nur eine ausführende oder teilnehmende Rolle hat. Für die strukturierte Inhaltsanalyse wurde das Softwarepaket ,MAXQDA Analytics Pro 2020 “ verwendet.

\section{Diskussion}

Die Studie basiert auf einer qualitativen inhaltlichen Analyse von Stellenangeboten für IT-Top-Management-Positionen (IT-Leiter, IT-Manager, IT-Leiter und CIO) in Deutschland, die 2020 (Februar und Oktober) zu zwei Zeitpunkten erhoben wurden.

\subsection{Hypothese und Forschungsfrage}

Die Hypothese für diese Forschung lautet:

Es gibt einen Einfluss der wirtschaftlichen und sozialen Auswirkungen der Covid19 Pandemie auf die Aufgaben eines IT-Top-Manager-Jobs.

Die Forschungsfrage lautet:

Werden Änderungen der IT-Prioritäten, die von Forschern und nicht wissenschaftlichen Autoren vorhergesagt wurden, 9 Monate nach Beginn der Pandemie in Stellenanzeigen widergespiegelt?

\subsection{Allgemeine Befunde (unabhängig von der Hypothese)}

Die Literaturrecherche in wissenschaftlichen Publikationen, Management-Zeitschriften sowie offiziellen und technischen Veröffentlichungen zeigt, dass der Gesamtfokus für IT-Abteilungen in dieser frühen Phase der Pandemie (derzeit 2. Welle in Deutschland) in der Bereitstellung von IT-Systemen zu liegen scheint. Diese unterstützen die Zusammenarbeit und das Arbeiten im Home Office (Shen et al. 2020; Bates 2020) sowie die Möglichkeit in etablierten Geschäftsprozessen zu arbeiten, ohne in der Firma anwesend zu sein. Informationssicherheit wird von Unternehmensberatern als sehr wichtig angesehen (PricewaterhouseCoopers 2020a; Shen et al. 2020; Bates 2020). Das Bundesamt für Sicherheit in der Informationstechnik (BSI) hat für 2020 einen Cybersicherheitsbericht veröffentlicht, welcher 


\section{JOB TITEL VERTEILUNG}

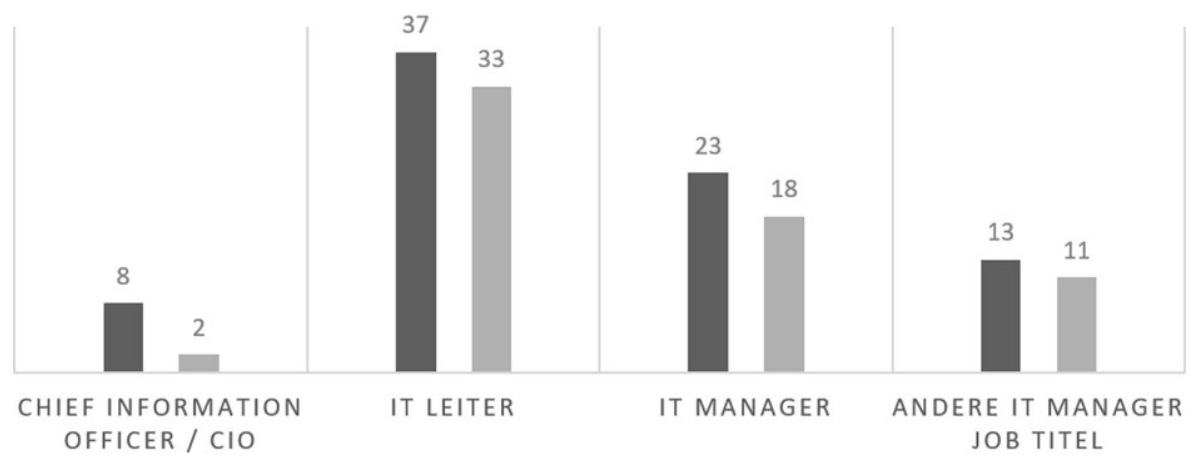

18. Feb. 2020 28. Oct. 2020

Abb. 2 Verteilung der Berufsbezeichnungen in der Datenerfassung. (Quelle: eigene Darstellung)

Aspekte und Warnungen in Zusammenhang mit COVID-19 beschreibt. Die Vorhersagen und Warnungen haben sich teilweise bewahrheitet und können in der Presse nachverfolgt werden. Offizielle Statistiken werden jedoch erst im nächsten Jahr im „Bericht über den Stand der IT-Sicherheit in Deutschland 2021“ veröffentlicht.

Die gesammelten Datensätze konnten in 4 Jobtitelgruppen zusammengefasst werden, wie in Abb. 2 ersichtlich. Einer der in den Stellenanzeigen verwendeten Jobtitel war eine Kombination aus mehreren Titeln. Um eine Doppeltzählung zu vermeiden, wurde nur einer der Jobtitel verwendet.

Die Stellenanzeigen wurden von Unternehmen aus nahezu allen Bundesländern Deutschlands veröffentlicht (siehe Tab. 2).

Auch wenn die Anzahl der nutzbaren Stellenanzeigen in dieser Studie um 20\% gesunken ist, ist die Tendenz bei der Verteilung von Berufsbezeichnungen und bei der regionalen Verteilung ähnlich. Ich komme daher zu dem Schluss, dass die Daten für die vorgeschlagene Forschungsfrage verglichen werden können.

\subsection{Vergleich der Ergebnisse mit Literaturmodellen und neuen IT-Manager- Aufgaben}

Krcmar entwickelte auf der Grundlage der Ergebnisse von Wollnik (1988) und Szyperski und Winand (1989) sowie seiner eigenen Forschung (Krcmar 1991) ein dreistufiges Modell des Informationsmanagements. Wollniks 3-Ebenen des Informationsmanagements (IM) sind gut etabliert, was auch durch die Erwähnungen in den Stellenanzeigen bezüglich des Managements von Anwendungen (52) und des Managements von Infrastruktur (66) gezeigt werden kann. Das Management von Informationen scheint jedoch von Personalabteilungen nicht als Aufgabe für ITManager angesehen zu werden, da sie nur spärlich erwähnt werden (6). Interessanterweise wird die vierte Aufgabe, die im Krcmars-Modell „Managementrolle der ITOrganisation“ bezeichnet wird, von Arbeitgebern nicht einheitlich gesehen. Mit der Folge, dass große Abweichungen bei den einzelnen Aufgaben gemessen wurden. Die vorgeschlagenen Unteraufgaben von Krcmar sind alle in der Gesamtheit der ge- 
Tab. 2 Regionale Verteilung von Stellenanzeigen. (Quellen: Februar 2020: indeed.com \& linkedin.com; Oct. 2020: indeed; eigene Berechnungen auf Basis der strukturierten Inhaltsanalyse)

\begin{tabular}{llll}
\hline Bundesländer & \multicolumn{2}{l}{ Regionale Verteilung der Anzeigen } \\
& $\begin{array}{l}18.02 .2020 \\
(n=79)\end{array}$ & $\begin{array}{l}28.10 .2020 \\
(n=63)\end{array}$ & Veränderung in \% \\
\hline Baden-Württemberg & $23,8 \%$ & $17,5 \%$ & $-6,3$ \\
Bayern & $13,8 \%$ & $17,5 \%$ & 3,7 \\
Berlin & $5,0 \%$ & $7,9 \%$ & 2,9 \\
Brandenburg & $1,3 \%$ & $0,0 \%$ & $-1,3$ \\
Bremen & $1,3 \%$ & $6,3 \%$ & 5,1 \\
Hamburg & $3,8 \%$ & $1,6 \%$ & $-2,2$ \\
Hessen & $10,0 \%$ & $12,7 \%$ & 2,7 \\
Mecklenburg-Vorpom- & $0,0 \%$ & $0,0 \%$ & 0,0 \\
mern & & & \\
Niedersachsen & $6,3 \%$ & $6,3 \%$ & 0,1 \\
Nordrhein-Westfahlen & $15,0 \%$ & $15,9 \%$ & 0,9 \\
Rheinland-Pfalz & $6,3 \%$ & $3,2 \%$ & $-3,1$ \\
Saarland & $0,0 \%$ & $0,0 \%$ & 0,0 \\
Sachsen & $5,0 \%$ & $4,8 \%$ & $-0,2$ \\
Sachsen-Anhalt & $3,8 \%$ & $0,0 \%$ & $-3,8$ \\
Schleswig-Holstein & $3,8 \%$ & $3,2 \%$ & $-0,6$ \\
Thüringen & $1,3 \%$ & $3,2 \%$ & 1,9 \\
Gesamtveränderung & $n=79$ & $n=63$ & $-20,3$ \\
\hline & & &
\end{tabular}

sammelten Stellenanzeigen erwähnt - jedoch mit mittlerer bis niedriger Frequenz ( 7 bis 37). Alle Häufigkeiten sind in Abb. 3 dargestellt und basieren auf dem Datensatz vom 18.02.2021.

Frühere Untersuchungen (Roscher und Brink 2020) haben gezeigt, dass Krcmars Modell um weitere Aufgaben erweitert werden sollte. Diese werden in Abb. 3 unter „NEUE Aufgaben“ aufgeführt. Dazu gehören die Bereiche Lieferantenkoordination und IT-Einkauf, Budgetierung, IT-Architekturmanagement, Projektmanagement sowie Beratung/Demand Management auf Management-Ebene.

\subsection{Spezifische Befunde zum Einfluss von COVID-19}

Wie im Abschnitt zur Codierung beschrieben, wurden alle Stellenanzeigen aus beiden Datenerhebungen nach Phrasen durchsucht, und für jede Fundstelle wurde eine manuelle Überprüfung durchgeführt. Anschließend erfolgte die Zählung. Die Ergebnisse werden in Tab. 3 dargestellt.

Diese qualitative Methode ist abhängig von der Kenntnis des IT-ManagementWortschatzes und IT-Prozesse. Aufgrund der geringen Anzahl von Daten in beiden Datensätzen und des manuellen Klassifizierungsprozesses der gefundenen Suchphrasen ist der Autor der Ansicht, dass die Ergebnisse einen Hinweis auf die Änderung des Inhalts von Jobzusätzen geben können, eine statistische Validität der Ergebnisse ist jedoch fraglich. 
Aufgaben einer IT Organisation basierend auf Stellenanzeigen gesammelt am 18. Feb. 2020
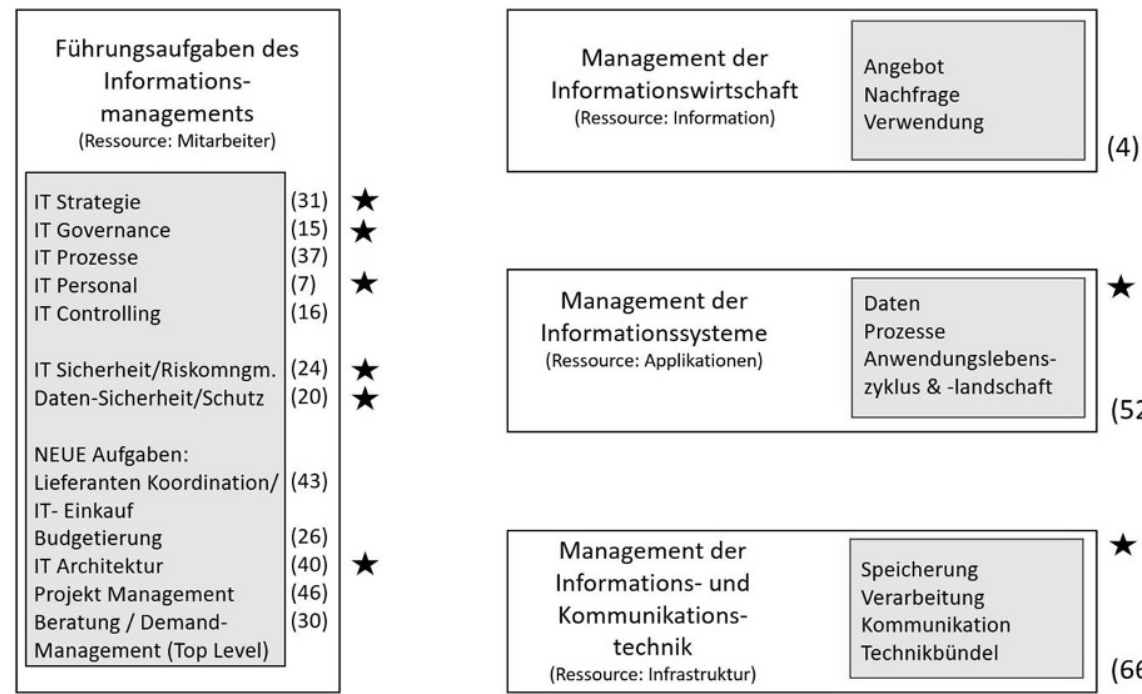

Firmen ( $\mathrm{N}=79$ );

Zahlen in (Klammern) in dieser Abbildung zeigen die Nennungen in den Stellenanzeigen;

$\star$ Bezeichnet die Aufgaben welche durch den Coding Prozess identifiziert wurden (siehe Tab. 1)

Abb. 3 Aufgaben von IT-Organisationen im Datensatz vor COVID-19. (Quelle: in Anlehnung an Krcmar (2015) mit Ergebnissen aus eigener Forschung)

Interessanterweise taucht die in Tab. 1 identifizierte höchste Priorität „,Digitale Zusammenarbeit“, welche in der Literatur mit Aufgaben wie der Bereitstellung von Infrastruktur und Anwendungen in Zusammenhang gebracht wird, im Aufgabenteil der Stellenanzeigen nicht auf.

Da jedoch auch IT-Manager solche digitalen Angebote nutzen und in dieser Zeit der COVID-19-Pandemie dem gleichen gesundheitlichen Risiko ausgesetzt sind wie alle anderen Mitarbeiter, enthalten die Stellenangebote Informationen über das zukünftige Arbeitsumfeld der IT-Top-Management-Kandidaten. Diese sind in Tab. 4 gezeigt.

Den Stellenanzeigen aus der Datensammlung vom 28. Oktober 2020 konnten bis auf eine Ausnahme ein Alter zugeordnet werden. Die Verteilung ist aus dem Histogramm in Abb. 4 ersichtlich. Zwei Nennungen aus der Kategorie „Homeoffice - nicht möglich“" waren älter als 29 Tage sowie eine Nennung aus der Kategorie „Remote - Nein“.

\section{Schlussfolgerung, Vorschläge, Empfehlungen}

Nach dem Vergleich der codierten Schlagwörter in beiden Datensätzen ist in der Analyse keine signifikante Änderung der Anforderungen von Aufgaben oder der Struktur der Stellenanzeigen festzustellen. Dies führt zu der Schlussfolgerung, dass die Personalabteilungen in Unternehmen veränderte Rahmenbedingungen entweder 
Tab. 3 Forschungsergebnisse zu den Aufgaben des IT-Managers. (Quelle: eigene Berechnungen auf Basis der strukturierten Inhaltsanalyse)

\begin{tabular}{|c|c|c|c|c|}
\hline $\begin{array}{l}\text { Literatur } \\
\text { Review Rang } \\
\text { (Nennungen) }\end{array}$ & $\begin{array}{l}\text { CIO Prioritäten } \\
\text { während und nach } \\
\text { COVID-19 }\end{array}$ & $\begin{array}{l}\text { Relative Nennungen } \\
\text { am } 18 \text {. Feb. } \\
\text { (basierend auf } \\
n=79 \text { ) in } \%\end{array}$ & $\begin{array}{l}\text { Relative Nennungen } \\
\text { am } 28 \text {. Okt. } \\
\text { (basierend auf } \\
n=63 \text { ) in } \%\end{array}$ & $\begin{array}{l}\text { Veränderung } \\
\text { in } \%\end{array}$ \\
\hline 1. $(n=4)$ & $\begin{array}{l}\text { Digitale Zusam- } \\
\text { menarbeit }\end{array}$ & Siehe Tab. 4 & & \\
\hline 2. $(n=3)$ & Informationssicherheit & 60,8 & 57,1 & $-3,6$ \\
\hline 2. $(n=3)$ & $\begin{array}{l}\text { Digitalisierung des } \\
\text { Geschäftsmodells }\end{array}$ & 1,3 & 1,6 & 0,3 \\
\hline 2. $(n=3)$ & $\begin{array}{l}\text { IT-Strategie und } \\
\text { Architektur }\end{array}$ & 29,1 & 22,2 & $-6,9$ \\
\hline 3. $(n=2)$ & $\begin{array}{l}\text { Weiterentwicklung } \\
\text { der digitalen Kun- } \\
\text { denschnittstelle }\end{array}$ & 7,6 & 11,1 & 3,5 \\
\hline 4. $(n=1)$ & Daten Sicherheit & 17,7 & 12,7 & $-5,0$ \\
\hline 4. $(n=1)$ & $\begin{array}{l}\text { Sicherstellung des } \\
\text { Geschäftsbetriebes }\end{array}$ & 2,5 & 3,2 & 0,6 \\
\hline 4. $(n=1)$ & Automatisierung & 15,2 & 17,5 & 2,3 \\
\hline 4. $(n=1)$ & Risiko Management & 5,1 & 1,6 & $-3,5$ \\
\hline 4. $(n=1)$ & Governance & 3,8 & 4,8 & 1,0 \\
\hline 4. $(n=1)$ & $\begin{array}{l}\text { Arbeitskraft (Work- } \\
\text { force) }\end{array}$ & 0,0 & 3,2 & 3,2 \\
\hline 4. $(n=1)$ & $\begin{array}{l}\text { Kosten Kontrolle } \\
\text { (Cost Control) }\end{array}$ & 8,9 & 9,5 & 0,7 \\
\hline
\end{tabular}

Tab. 4 Forschungsergebnisse zum persönlichen Arbeitsumfeld des IT-Top-Management Kandidaten. (Quelle: eigene Berechnungen auf Basis der strukturierten Inhaltsanalyse)

\begin{tabular}{llll}
\hline & $\begin{array}{l}\text { Relative Nennungen am } \\
18 . \text { Feb. } \\
\text { (basierend auf } n=79)\end{array}$ & $\begin{array}{l}\text { Relative Nennungen am } \% \\
28 . \text { Okt. } \\
\text { (basierend auf } n=63) \text { in } \%\end{array}$ & $\begin{array}{l}\text { Veränderung } \\
\text { in \% }\end{array}$ \\
\hline $\begin{array}{l}\text { Homeoffice - Standard } \\
\text { Homeoffice - temporär/ }\end{array}$ & 1,3 & 9,5 & 8,3 \\
wegen Covid-19 & 0,0 & 12,7 & 12,7 \\
$\begin{array}{l}\text { Homeoffice - nicht mög- } \\
\text { lich }\end{array}$ & 0,0 & 6,3 & 6,3 \\
$\begin{array}{l}\text { Remote - wenn notwendig } \\
\text { Remote - Nein }\end{array}$ & 1,3 & & $-1,3$ \\
\hline
\end{tabular}

gar nicht oder nur sehr zögerlich in Stellenanzeigen einarbeiten. Über die Gründe kann nur spekuliert werden und ist daher nicht Gegenstand dieser Arbeit.

Die einzigen Spuren, die Covid-19 in den Stellenanzeigen hinterlassen hat, sind einzelne Erwähnungen von Home-Office-Richtlinien.

Die Hypothese und die Forschungsfrage können nur durch die Literaturrecherche unterstützt werden, die strukturierte Inhaltsanalyse hingegen zeigt ausschließlich signifikante Ergebnisse im Bereich der Home-Office-Richtlinien. 
Abb. 4 Alter der Stellenanzeigen vom 28. Okt. 2020 in Tagen, aufgetragen in 10 Tages Intervallen. (Quelle: eigene Berechnungen auf Basis der strukturierten Inhaltsanalyse)

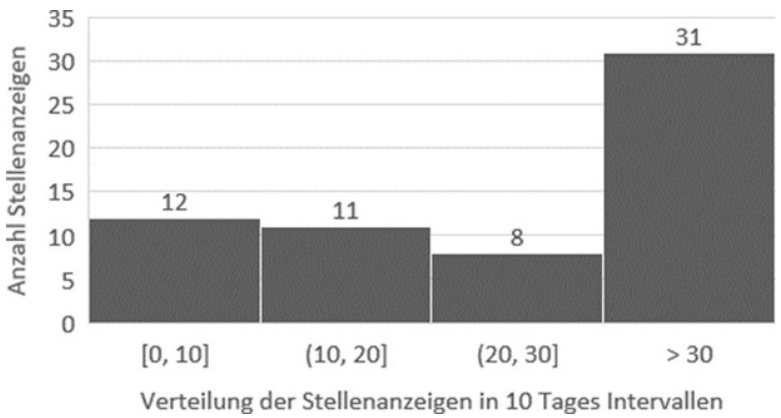

Die Hauptempfehlung an die Autoren von Stellenanzeigen für IT-Top-Management-Positionen besteht darin, aktuelle Herausforderungen in die Anforderungen der Stellenbeschreibung aufzunehmen. Es scheint, dass die meisten Stellenanzeigen auf „Copy and Paste“ basieren und dann entweder inhaltlich angereichert oder reduziert werden. Nur wenige zeichnen ein Bild über die Einflussmöglichkeiten und Chancen einer Position. Das Vorhandensein würde aber einen entscheidenden Vorteil für die Suche nach der richtigen Person für die offene Position bringen, besonders in einem von Kandidaten geprägten IT-Arbeitsmarkt. Im Zuge des Self-Selection-Prozesses der in dem Principal-Agent-Model (Eisenhardt 1989) beschrieben wird, wäre dies für eine erfolgreiche Besetzung der offenen Stellen vorteilhaft.

Zukünftige Forschung sollten mehr Daten erfassen, um eine höhere statistische Relevanz zu erzielen. Die Automatisierung der Klassifizierung von Suchergebnissen könnte dann dazu beitragen, den Codierungs-, Bewertungs- und Berechnungsprozess zu beschleunigen. Die vorgestellte Methode könnte einen Beitrag zur nachträglichen Analyse von Änderungen der Unternehmensumwelt und der Auswirkungen auf bestimmte Jobprofile im Zeitverlauf leisten.

\section{Literatur}

Barak I (2020) Critical infrastructure under attack. Lessons from a honeypot. Netw Secur 2020(9):16-17 Bates S (2020) COVID-19. Insights for CIOs and IT executives. https://advisory.kpmg.us/insights/insightsfor-your-role/cio-real-insights/covid-19-responses-it-executives.html. Zugegriffen: 31. Okt. 2020

Bundesamt für Sicherheit in der Informationstechnik (BSI) (2020) Die Lage der IT-Sicherheit in Deutschland. https://www.bsi.bund.de/SharedDocs/Downloads/DE/BSI/Publikationen/Lageberichte/ Lagebericht2020.pdf?_blob=publicationFile\&v=2. Zugegriffen: 29. Okt. 2020

Chan YE, Sabherwal R, Thatcher JB (2006) Antecedents and outcomes of strategic IS alignment. An empirical investigation. IEEE Trans Eng Manag 53(1):27-47

Chen C-F, Zarazua de Rubens G, Xu X, Li J (2020) Coronavirus comes home? Energy use, home energy management, and the social-psychological factors of COVID-19. Energy Res Soc Sci 68:101688

Deloitte Insights (2020) Digital transformation through the lens of COVID-19. https://www2.deloitte. com/us/en/insights/topics/digital-transformation/digital-transformation-COVID-19.html. Zugegriffen: 21. Okt. 2020

Durst M (2007) Wertorientiertes Management von IT-Architekturen. Teubner, Wiesbaden

Dwivedi YK, Hughes DL, Coombs C, Constantiou I, Duan Y, Edwards JS, Gupta B, Lal B, Misra S, Prashant P, Raman R, Rana NP, Sharma SK, Upadhyay N (2020) Impact of COVID-19 pandemic on information management research and practice. Transforming education, work and life. Int $\mathrm{J}$ Inf Manag 55:102211

Eisenhardt KM (1989) Agency theory. An assessment and review. Acad Manag Rev 14(1):57 
Faulds DJ, Raju PS (2020) The work-from-home trend. An interview with Brian Kropp. Bus Horiz. https:// doi.org/10.1016/j.bushor.2020.10.005

Furnell S, Shah JN (2020) Home working and cyber security-an outbreak of unpreparedness? Comput Fraud Secur 2020(8):6-12

Grohmann HH (2003) Prinzipien der IT-Governance. HMD Prax Wirtschaftsinf 40(232):17-23

Hodgkinson SL (1992) IT structures for the 1990s:. organisation of IT functions in large companies. A survey. Inf Manag 22(3):161-175

IDG (2020) CIO COVID-19 impact study. https://www.idg.com/tools-for-marketers/cio-cv-19-impactstudy/. Zugegriffen: 31. Okt. 2020

Kim M-K, Jee K-Y (2007) Factors influencing strategic use of information technology and its impact on business performance of SMEs. ETRI J 29(4):497-506

King WR, Grover V, Hufnagel EH (1989) Using information and information technology for sustainable competitive advantage. Some empirical evidence. Inf Manag 17(2):87-93

Krcmar H (1991) Integration in der Wirtschaftsinformatik - Aspekte und Tendenzen. Integrierte Informationssysteme. Gabler, Wiesbaden, S 190

Krcmar H (2015) Informationsmanagement. Springer Gabler, Berlin, Heidelberg, S 107

Kumar A, Luthra S, Mangla SK, Kazançoğlu Y (2020a) COVID-19 impact on sustainable production and operations management. Sustain Oper Comput 1:1-7

Kumar S, Raut RD, Narwane VS, Narkhede BE (2020b) Applications of industry 4.0 to overcome the COVID-19 operational challenges. Diabetes Metab Syndr 14(5):1283-1289

Malecki F (2020) Overcoming the security risks of remote working. Comput Fraud Secur 2020(7):10-12

Marrone M, Kolbe LM (2011) Einfluss von IT-Service-Management-Frameworks auf die IT-Organisation. Wirtschaftsinformatik 53(1):5-19

Mayring P (2015) Qualitative Inhaltsanalyse. Grundlagen und Techniken. Beltz, Weinheim

Meiler Y (2020) Digital transformation, covid-19 crisis, digital transformation. In: Bunkanwanicha P, Coeurderoy R, Ben Slimane S (Hrsg) Managing a post-covid19 era. ESCP impact papers. ESCP Research Institute of Management (ERIM), Paris, S 171-178

Moher D, Shamseer L, Clarke M, Ghersi D, Liberati A, Petticrew M, Shekelle P, Stewart LA (2015) Preferred reporting items for systematic review and meta-analysis protocols (PRISMA-P) 2015 statement. Syst Rev. https://doi.org/10.1186/2046-4053-4-1

Mouton F, Coning A (2020) Covid-19: impact on the cyber security threat landscape. https://www. researchgate.net/publication/340066124_COVID-19_Impact_on_the_Cyber_Security_Threat_ Landscape. Zugegriffen: 28. Okt. 2020

Peppard J (2010) Unlocking the performance of the chief information officer (CIO). Calif Manage Rev 52(4):73-99

PricewaterhouseCoopers (2020a) How technology leaders are responding to the COVID-19 crisis. https://www.strategyand.pwc.com/de/de/implications- of-covid-19/how-technology-leaders-areresponding-to-the-covid-19-crisis.html. Zugegriffen: 31. Okt. 2020

PricewaterhouseCoopers (2020b) How technology leaders are responding to the COVID-19 crisis. https://www.strategyand.pwc.com/de/de/implications-of-covid-19/how-technology-leaders-areresponding-to-the-covid-19-crisis.html. Zugegriffen: 31. Okt. 2020

Roscher BE (2020) Impact of the COVID-19 Crisis on the Structure and Content of Job Advertisements. In: Proceedings of the 3rd International Conference on Research in Business, Management and Economics. (ICRBME) Dublin, Republic of Ireland

Roscher BE, Brink N (2020) Tasks of IT managers: empirical research based on job advertisements in Germany. In: Proceedings of the 3rd International Conference on Applied Research in Management, Economics and Accounting IARMEA, Paris, France

Rüter A, Schröder J, Göldner A, Niebuhr J (2010) IT-Governance in der Praxis. Erfolgreiche Positionierung der IT im Unternehmen. Anleitung zur erfolgreichen Umsetzung regulatorischer und wettbewerbsbedingter Anforderungen. Springer, Berlin, Heidelberg

Samuels M (2020) The CIO has a new set of priorities and problems. Here's what's on their to-do list. https://www.zdnet.com/article/the-cio-has-a-new-set-of-priorities-and-problems-heres-whats-ontheir-to-do-list/. Zugegriffen: 31. Okt. 2020

Schweda CM, Schmidt W (2014) Leichtgewichtiges, unternehmensspezifisches IT-Management. HMD Prax Wirtschaftsinf 51(5):606-615

Shen S, Chen O, Sun J, Mok L, Gao A, Deacon DKW (2020) Coronavirus (COVID-19) outbreak: shortand long-term actions for CIOs. CIOs need to increase resilience against future disruptions and prepare for rebound and growth. https://www.gartner.com/en/doc/720647-covid-19-outbreak-short-andlong-term-actions-for-cios. Zugegriffen: 31. Okt. 2020 
Synnott WR, Gruber WH (1981) Information resource management. Opportunities and strategies for the 1980s. Wiley, New York

Szyperski N, Winand U (1989) Informationsmanagement und informationstechnische Perspektiven. In: Bleicher K, Seidel E, Wagner D (Hrsg) Organisation. Evolutionäre Interdependenzen von Kultur und Struktur der Unternehmung: Knut Bleicher zum 60. Geburtstag. Gabler, Wiesbaden, S 133-150

Tallon PP (2014) A process-oriented perspective on the alignment of information technology and business strategy. J Manag Inf Syst 24(3):227-268

Ward J, Peppard J (2002) Strategic planning for information systems. John Wiley \& Sons, Chichester

Weill P, Ross JW (2004) IT governance: how top performers manage IT decision rights for superior results. Int J Electron Gov Res 1(4):63-67

Weill P, Woodham R (2002) Don't just lead, govern. Implementing effective IT governance. SSRN J. https://doi.org/10.2139/ssrn.317319

Wollnik M (1988) Ein Referenzmodell des Informationsmanagements. Inf Manag 3(3):34-43

Zellmer G (1990) Risiko-Management. Die Wirtschaft, Berlin 